\title{
The Potential Utility of Aripiprazole Augmentation for Major Depressive Disorder with Mixed Features Specifier: A Retrospective Study
}

\author{
Changsu Han ${ }^{1}$, Sheng-Min Wang ${ }^{2,3}$, Won-Myong Bahk ${ }^{2}$, Soo-Jung Lee ${ }^{2}$, Ashwin A. Patkar ${ }^{4}$, Prakash S. Masand ${ }^{5}$, \\ Chi-Un Pae ${ }^{2,4,6}$ \\ ${ }^{1}$ Department of Psychiatry, Korea University College of Medicine, ${ }^{2}$ Department of Psychiatry, College of Medicine, The Catholic University \\ of Korea, ${ }^{3}$ International Health Care Center, Seoul St. Mary's Hospital, College of Medicine, The Catholic University of Korea, Seoul, Korea, \\ ${ }^{4}$ Department of Psychiatry and Behavioral Sciences, Duke University Medical Center, Durham, NC, ${ }^{5}$ Global Medical Education, New York, \\ NY, USA, ${ }^{6}$ Cell Death Disease Research Center, College of Medicine, The Catholic University of Korea, Seoul, Korea
}

\begin{abstract}
Objective: The present study aimed to observe potential benefit of aripiprazole augmentation in the treatment of major depressive disorder with mixed specifier (MDDM) in naturalistic treatment setting.

Methods: Data were collected from MDDM patients using a retrospective chart review for 8 weeks (week -8 and week 0) in routine practice. All patients were on current antidepressants upon starting of aripiprazole. Patients were treated without restriction of doses of aripiprazole. The primary endpoint was the mean change of Montgomery- $\AA_{s b e r g}$ Depression Rating Scale (MADRS) total scores along with various secondary endpoint measures.

Results: In total 38 patients were analyzed. The changes of MADRS, Clinical Global Impression (CGI)-severity, Young Mania Rating Scale, Sheehan Disability Scale, and CGl-clinical benefit total scores from baseline to the endpoint were $-7.1,-0.8,-4.9,-4.1$, and -3.6 , respectively (all $p<0.0001$ ). At the endpoint, the responder and remitter rates by MADRS score criteria were approximately $32 \%$ and $21 \%$, respectively.

Conclusion: The present findings have clearly shown the effectiveness and tolerability of aripiprazole augmentation for MDDM patients in routine practice. The present study warrants subsequent, adequately-powered, well-controlled studies for generalizability near future.
\end{abstract}

KEY WORDS: Aripiprazole; Depressive disorder; Mixed specifier; Effectiveness; Tolerability.

\section{INTRODUCTION}

The ideal goal of antidepressant treatment for patients with major depressive disorder (MDD) is to achieve full recovery of individual, occupational, and psychosocial functions. However, lower remission/response rates and higher relapse rates have been consistently reported in real world treatment setting [1-3]. In addition, some subpopulations of MDD including mixed or anxious features

Received: October 21, 2018 / Revised: December 19, 2018 Accepted: January 23, 2019

Address for correspondence: Chi-Un Pae

Department of Psychiatry, Bucheon St. Mary's Hospital, College of Medicine, The Catholic University of Korea, Sosaro 327,

Wonmi-gu, Bucheon 14647, Korea

E-mail: pae@catholic.ac.kr

ORCID: https://orcid.org/0000-0003-1632-4248 are known to present poor clinical outcomes than those without such subsymptoms [4].

Likewise, the literature shows 'mixed feature' are present approximately in $30 \%$ of patients with MDD depending on methodological approach [5-7]. These symptoms can significantly increase risk for suicidal behaviour, result in poor antidepressant treatment response, substance abuse, relapse/recurrence, and (hypo)manic switch in patients with MDD [5]. Aripiprazole has been widely accepted and used for the treatment of MDD in clinical practice today; however, there has been no such information on the use of aripiprazole for the treatment of MDD with mixed specifier (MDDM) yet [8.9].

The pharmacological characteristics and proven augmentation efficacy of aripiprazole for MDD warrant its use for the treatment of MDDM. Aripiprazole's partial ag-

(c) This is an Open-Access article distributed under the terms of the Creative Commons Attribution Non-Commercial License (http://creativecommons.org/licenses/by-nc/4.0) which permits unrestricted non-commercial use, distribution, and reproduction in any medium, provided the original work is properly cited. 
onist effects on D2/D3, 5-HT and 5-HT1A as well as its antagonist effects on 5-HT6, 5-HT7 and 5-HT2C receptors, leading to alteration of dopamine, serotonin and norepinephrine in specific brain areas have been considered in favour of improvement of mood symptoms [10]. In addition aripiprazole has shown substantial improvement of variation in mood, impulsivity, agitation, anxiety and irritability which are commonly seen in MDDM [11]. Aripiprazole has shown potential as proper augmentation agent for patients with MDD regardless of such subsymptoms including other clinical factors according to recent researches [12-14]. In addition, there has been a putative hypothesis proposing some clinical similarities between MDDM, neuroleptic-induced dysphoria, withdrawal syndromes from dopaminergic drugs, and other behavioural syndromes arising from sudden dopamine depletion [15]. In this context, recent positron emission tomography (PET) study has revealed the main effects of aripiprazole would be produced by potentiation of dopaminergic activity [16]. In the study, 14 MDD patients, who had failed 8 weeks of antidepressant therapy with selective serotonin reuptake inhibitors, underwent 6-[18F]-L-DOPA (F-DOPA) PET scans before and after aripiprazole treatment; 11 responded to aripiprazole augmentation. Whole brain, voxel-wise comparisons of pre- and post-aripiprazole scans revealed increased F-DOPA trapping in the right medial caudate of aripiprazole augmentation responders [16]. An exploratory analysis of depressive symptoms revealed that such responders experienced large improvements only in putatively dopaminergic symptoms of lassitude and inability to feel [16].

Given above findings, we may assume that aripiprazole could be a potential agent as an augmentation therapy for treatment of MDDM patients. This retrospective study is thus proposed to observe how well aripiprazole works to treat MDDM in routine practice and provide a bridging data expanding to future double-blind randomized clinical trials (RCTs).

\section{METHODS}

This study aimed to observe the potential utility of aripiprazole for treatment of MDDM (the Diagnostic and Statistical Manual of Mental Disorders 5th edition, DSM-5) [17] patients who have shown inadequate re- sponse to ongoing antidepressant treatment in routine clinical practice. The study was conducted in two university-based teaching hospitals. The MDDM diagnosis was confirmed when the patient met the full criteria of MDD episode and had simultaneously at least three of the following manic/hypomanic symptoms defined as DSM-5 (e.g., elevated and expansive mood, inflated self-esteem, talkativeness, flight of ideas, increased energy, increased or excessive involvement in activities that have a high potential for painful consequences, and decreased need for sleep) during the majority of days during the current or most recent episode of MDD.

All data collection was done for those who were treated with aripiprazole to treat symptoms of MDDM in routine outpatient practice for 8 weeks (week -8 , baseline; week 0 , endpoint). Aripiprazole was not intentionally used for study purpose; the treatment was completely based on clinical purpose and clinicians' proper judgment that the patients may have clinical benefits with the use of aripiprazole. Aripiprazole was added on current antidepressant without dose restriction. The inclusion criteria are as follows: patients with MDDM, patients with inadequate antidepressant response (less than 50\% improvement after at least 6-week antidepressant treatment), and patients with aripiprazole treatment to augment current antidepressant effect. Patients having other than MDD, other antipsychotics, mood stabilizers, psychostimulants, and change of antidepressant during observation period were excluded.

The primary efficacy was the mean change of Montgomery - Åsberg Depression Rating Scale (MADRS) [18] total scores from week -8 to week 0 and the secondary measures were mean changes in the Clinical Global Impression-Severity (CGI-S), Young Mania Rating Scale (YMRS) [19], Sheehan Disability scale (SDS) [20], and CGl-Clinical Benefit (CGI-CB) [21] total scores. Proportions of responder ( $\geq 50 \%$ reduction of MADRS total score at the end of treatment) and remitter ( $\leq 10$ of MADRS total score at the end of treatment) were also collected as well as proportion of patients achieving improvement in CGI-CB score at the end of treatment to measure clinical benefit. CGI-CB score of 1 indicates the greatest benefit (effectiveness) with least burden (adverse events, AEs) from treatment, whereas score of 10 indicates the least benefit with the greatest burden from the treatment. The proportion of patients achieving 1 or 2 
scores of CGI- Improvement at the end of treatment was also collected. Any occurrence of AEs throughout the study was reported.

The sample size of the present study was more than $95 \%$, with observed mean difference in MADRS at the end of treatment $(d=7.1$, standard deviation $=6.8)$, under an alpha value of 0.05 with two tailed. Analyses will include intent-to-treat for the end of treatment. Non-parametric analyses were done if the data distribution was not in normative range after Kolmogorov - Smirnov test, otherwise all data was analyzed by parametric analyses. Descriptive statistics, Wilcoxon signed rank test (or paired $t$ test), chi-square test were performed where appropriate. Statistical significance was determined at $p<0.05$. Statistical analysis was performed using the NCSS ver. 07 for Windows (NCSS Inc., Kaysville, UT, USA) program.

The present study has been thoroughly reviewed and approved by local Institutional Review Board (approval number: HC16RIMI0112), and was conducted in compliance with the Declaration of Helsinki.

\section{RESULTS}

Thirty-eight patients were enrolled and analyzed during the study. The sample included a greater proportion of male patients $(65.8 \%)$ with approximately 28 years old. The mean duration of MDD was about 4 years. The baseline MADRS and YMRS total scores were approximately 22 and 9, respectively. The most common antidepressant and antianxiety medication were escitalopram and lorazepam, respectively. The mean aripiprazole dose was 4.0 ( \pm 0.8$) \mathrm{mg} /$ day during the study. Table 1 displays baseline clinical and demographical of patients in detail.

The changes of MADRS, CGI-S, YMRS, SDS, and CGI-CB total scores from baseline to the endpoint were $-7.1,-0.8,-4.9,-4.1$, and -3.6 , respectively (all $p<$ 0.0001 ) (Fig. 1). Each assessment score has decreased by $31.9 \%, 19.5 \%, 55.1 \%, 23.6 \%$, and $45.0 \%$, respectively from baseline to the endpoint.

At the endpoint, the responder and remitter rates by MADRS score criteria were approximately $32 \%$ and $21 \%$, respectively (Fig. 2). The responder rates by $\mathrm{CGI}$ and YMRS score criteria were approximately $47 \%$ and $61 \%$, respectively, at the endpoint (Fig. 3).

In total, approximately $79 \%$ of patients $(n=30)$ showed a decrease in CGI-CB score at the endpoint. In detail, The
Table 1 . Summary of baseline characteristics of the subjects $(n=38)$

\begin{tabular}{|c|c|}
\hline Parameter & Data \\
\hline Age (yr) & $28.4 \pm 11.3$ \\
\hline Sex, male & 25 (65.8) \\
\hline Duration of illness (yr) & $4.4 \pm 2.9$ \\
\hline Number of MDD episode & $1.9 \pm 0.8$ \\
\hline Duration of current MDD episode (mo) & $8.5 \pm 7.6$ \\
\hline Number of admission & $0.1(0.2)$ \\
\hline Presence of comorbid medical illness & $13(34.2)$ \\
\hline Presence of past history of admission & $10(26.3)$ \\
\hline Presence of family history & $5(13.2)$ \\
\hline \multicolumn{2}{|l|}{ Socioeconomic status } \\
\hline Middle & $27(71.1)$ \\
\hline Low & $10(26.3)$ \\
\hline Secured by government & $1(2.6)$ \\
\hline History of substance use (alcohol/nicotine) & $5(13.2)$ \\
\hline Living status, married & $7(18.4)$ \\
\hline \multicolumn{2}{|l|}{ Education } \\
\hline$\leq$ Middle school & $4(10.5)$ \\
\hline$\geq$ High school & $34(89.5)$ \\
\hline \multicolumn{2}{|l|}{ Type of antidepressants } \\
\hline \multicolumn{2}{|l|}{ SSRIs } \\
\hline Escitalopram & $18(47.4)$ \\
\hline Fluoxetine & $9(23.7)$ \\
\hline Sertraline & $3(7.9)$ \\
\hline \multicolumn{2}{|l|}{ SNRIS } \\
\hline Duloxetine & $2(5.2)$ \\
\hline Venlafaxine & $5(13.2)$ \\
\hline \multicolumn{2}{|l|}{ DNRI } \\
\hline Bupropion & $1(2.6)$ \\
\hline \multicolumn{2}{|l|}{ Concomitant anti-anxiety drugs } \\
\hline Presence & $25(65.8)$ \\
\hline Alprazolam & $5(20.0)$ \\
\hline Lorazepam & $8(32.0)$ \\
\hline Buspiron & $5(20.0)$ \\
\hline Etizolam & $4(16.0)$ \\
\hline Others & $3(12.0)$ \\
\hline None & $13(34.2)$ \\
\hline \multicolumn{2}{|l|}{ Psychometirc assessment } \\
\hline CGI-CB & $8.0 \pm 2.4$ \\
\hline CGI-S & $4.1 \pm 2.6$ \\
\hline MADRS & $21.9 \pm 5.4$ \\
\hline YMRS & $8.9 \pm 4.6$ \\
\hline SDS & $17.4 \pm 5.2$ \\
\hline
\end{tabular}

Values are presented as mean \pm standard deviation or number (\%). MDD, major depressive disorder; CGI-CB, Clinical Global ImpressionClinical Benefit; CGI-S, Clinical Global Impression-Severity; MADRS, Montgomery - Åsberg Depression Rating Scale; YMRS, Young Mania Rating Scale; SDS, Sheehan Disability Scale; SSRI, selective serotonin reuptake inhibitors; SNRI, serotonin-norepinephrine reuptake inhibitor; DNRI, dopamine-norepinephrine reuptake inhibitor.

most frequent score at baseline on the CGI-CB was $10(\mathrm{n}=$ 15), indicating unchanged/worse in therapeutic effect and outweigh therapeutic effect in burden of AEs, followed by scores of $7(n=9), 8(n=7), 4(n=5)$ and $3(n=1) / 9(n$ 


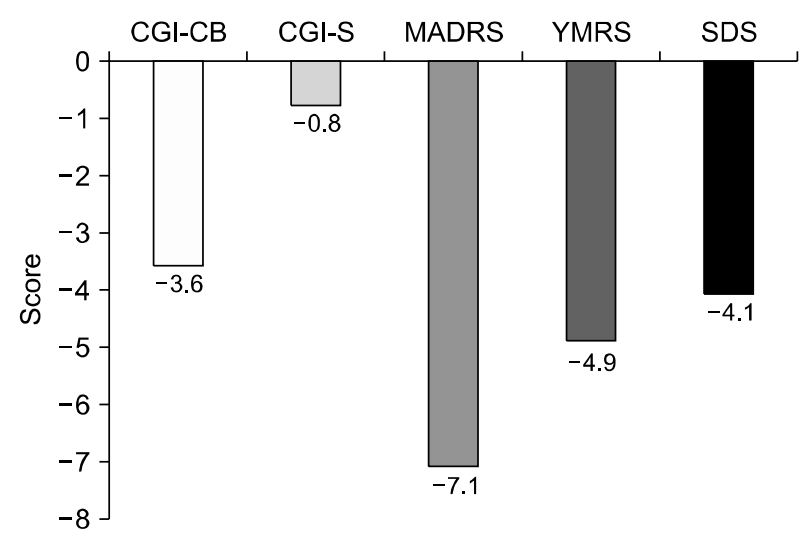

Fig. 1. Changes of outcome measures from baseline in the study. Data represent mean numbers from baseline at each psychometric scale.

CGI-CB, Clinical Global Impression-Clinical Benefit; CGI-S, Clinical Global Impression-Severity; MADRS, Montgomery - Åsberg Depression Rating Scale; YMRS, Young Mania Rating Scale; SDS, Sheehan Disability Scale.

All $p$ values $<0.001$.

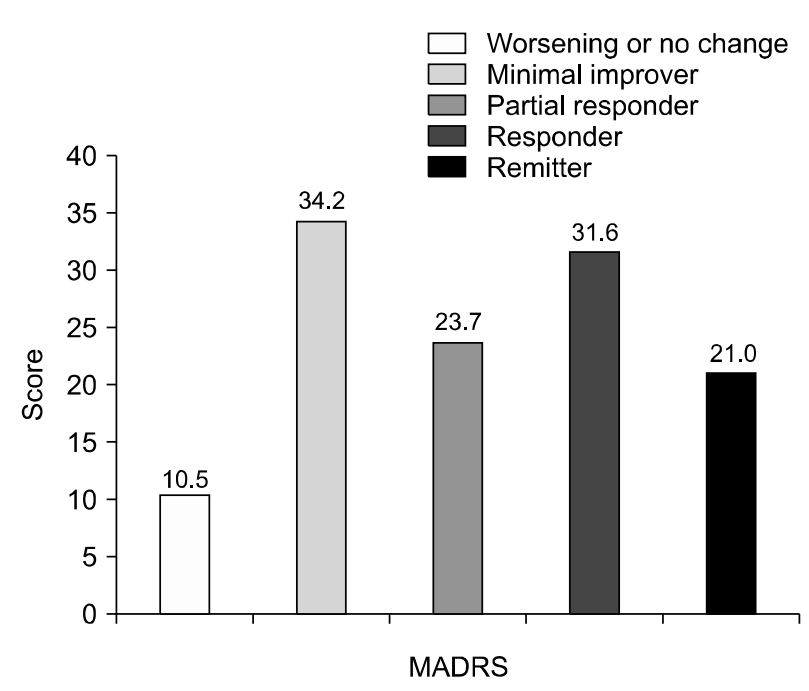

Fig. 2. Responder and remitter rates (\%) at the end of treatment in the study. Data represent percentage of subjects as indicated.

MADRS, Montgomery - Åsberg Depression Rating Scale; Worsening or no change, no change or increase of MADRS total score; minimal responder, $0 \%<$ decrease of MADRS total score $<25 \%$; partial responder, $25 \% \leq$ decrease of MADRS total score $<50 \%$; responder, $\geq 50 \%$ decrease of MADRS total score; remitter, $11 \leq$ MADRS total score at the end of treatment.

$=1$ ) indicating minimal to moderate therapeutic effect and none to significant interference in burden of AEs. At the endpoint, the most frequent scores at baseline on the CGI-CB were $4(n=10), 3(n=7)$, and $1(n=6)$ indicating moderate to marked therapeutic effect and none to no significant interference in burden of AEs, followed by scores

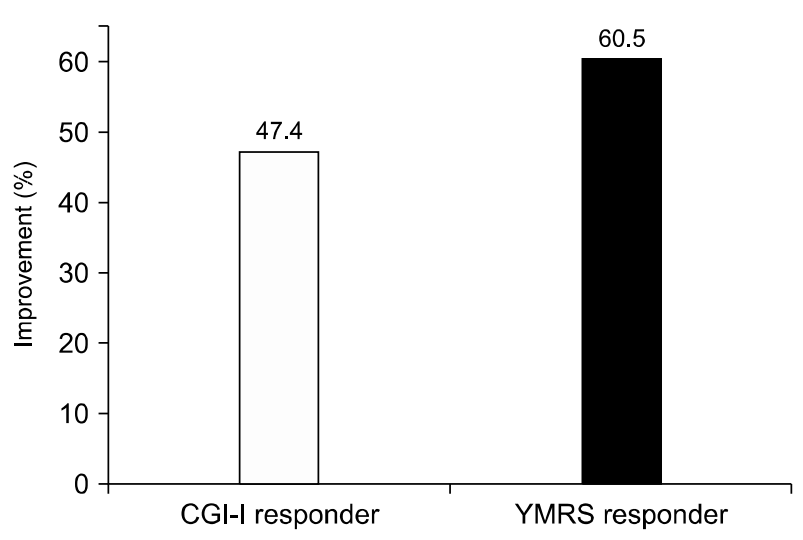

Fig. 3. Improvement rate by Clinical Global Impression-Improvement (CGI-I) score at the end of treatment.

YMRS, Young Mania Rating Scale; CGI-I responder, subject on score 1 or 2 of CGI-I score at the end of treatment; YMRS responder, $\geq 50 \%$ decrease of YMRS total score at the end of treatment.

Aripiprazole doses: $4.3 \pm 3.1 \mathrm{mg} /$ day and $3.9 \pm 2.5 \mathrm{mg} /$ day at CGI-I responders and YMRS responders, respectively.

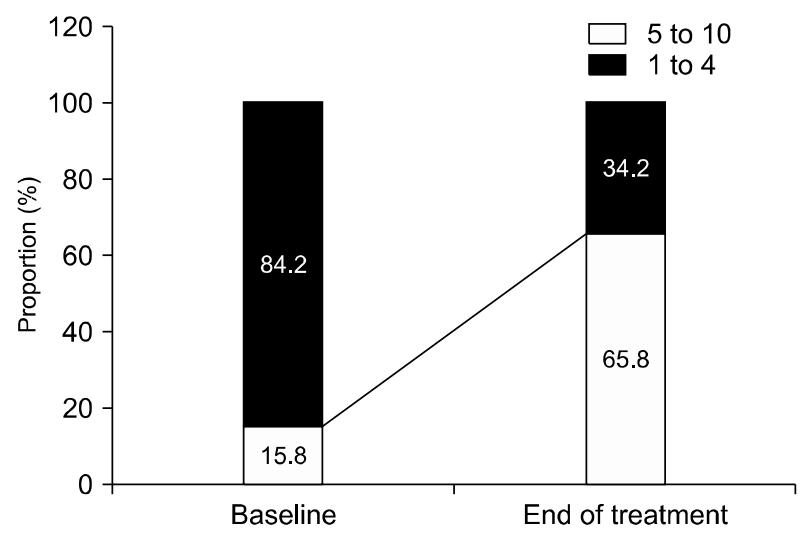

Fig. 4. Comparison of distribution of Clinical Global Impression-Clinical Benefit (CGI-CB) scores (1 to 10) between baseline and end of treatment. CGI-CB score of 1 indicates the greatest benefit (effectiveness) with least burden (adverse events) from treatment, whereas score of 10 indicates the least benefit with the greatest burden from the treatment.

of $7(n=7), 8(n=3), 2(n=2), 10(n=2)$, and $6(n=1)$ indicating unchanged/worse to marked therapeutic effect and none to outweigh therapeutic effect in burden of AEs. The CGI-CB improver rates scoring 1 to 4 (likely beneficial) or 5 to 10 (less likely beneficial) were $66 \%$ and $34 \%$, respectively (Fig. 4).

Increased appetite, somnolence, and headache were reported in only three patients during the study. There was no extrapyramidal symptoms (EPS) or movement-related AEs. The Barnes Akathisia Rating Scale and SimpsonAngus Rating total scores were zero with any changes 
during the study.

\section{DISCUSSION}

To the best of our knowledge, the present study is the first one to investigate the effects of aripiprazole augmentation for treatment of MDDM. The present study aimed to see the potential utility of aripiprazole augmentation for MDDM patients in naturalistic treatment setting and aripiprazole has clearly shown its clinical benefit as augmentation agents in the treatment of MDDM. According to the results, aripiprazole augmentation significantly improved the MADRS total score as well as all other secondary efficacy measures, without any significant AEs during the whole study period. Despite no official treatment guideline has been available for treatment of MDDM, Dr. Stahl group has recently suggested pharmacological treatment guideline proposing atypical antipsychotics including aripiprazole as the first-line treatment for MDDM [22].

The MADRS total score change was approximately 7 point which is comparable with those (about 10 points) from other RCTs and open-label studies. In addition, the responder rates by different criteria were approximately $30 \%$ to $60 \%$, indicating that proper treatment effects were observed in significant portion of patients. In addition, the remission rate was increased by $15.8 \%$ when applying MADRS score cut-off point of 11 as other researchers proposed [23].

Another intriguing point is that the reduction of YMRS total score was also quite comparable to that of MADRS and the responder rate by YMRS criteria was approximately two times higher than that by MADRS criteria. This is in line with the previously proven efficacy of aripiprazole for treatment of acute manic or mixed episodes of bipolar I disorder [24,25].

According to previous subanalyses of aripiprazole MDD RCTs, aripiprazole was proven to be effective regardless of subtype [12] or severity [13] of MDD, which is in line with our results. Indeed there have been no identified clinical factors (antidepressant type, doses, duration of illness, comorbidity, e.t.c) to be associated with the effects of aripiprazole augmentation in the treatment of MDD yet, which are consistently and similarly observed in subsequent RCTs [26] and subanalyes $[27,28]$.

Meanwhile, aripiprazole dose was low in our study, when reflecting the clinical manifestation of MDDM. This is contrary to what was expected but is supported by other successful clinical experiences of low-dose aripiprazole augmentation for refractory MDD in Asian populations. In addition, our dose $(4 \mathrm{mg} /$ day) is in dose range previously reported in MDD RCTs, open trials, and medical data analysis (3 to $12 \mathrm{mg} /$ day) $[26,27,29-36]$. Indeed, recent Japanese RCT [26] has clearly proven equally efficacy between low ( $3 \mathrm{mg} /$ day) and high dose $(10 \mathrm{mg} /$ day) in MDD. In addition, the most recent and largest observation study $(n=1,103)$ of aripiprazole augmentation for MDD has also reported low dose of $3.5 \mathrm{mg} /$ day used in routine practice. In a recent chart review study $(n=211)$, low doses of aripiprazole ( $\leq 5 \mathrm{mg}$ ), has been proposed to be more effective and better tolerated than higher ones in the treatment of bipolar II or bipolar not otherwise specified depression as well [37]. A recent bipolar depression RCTs using aripiprazole have failed to show its clear superiority over placebo; one of main reasons was the use of high dose resulting in high rate of early dropout due to EPS and other multiple AEs [38]. In addition in the first RCT using atypical antipsychotics (lurasidone) for MDDM [39], the dose of lurasidone $(36.2 \mathrm{mg} /$ day) was almost half dose of the used in bipolar depression $(64.6 \mathrm{mg} /$ day) RCT of lurasidone [40]. Finally, the effects of the partial agonist effects of aripiprazole on presynaptic dopamine synthesis in relation to dopamine D2 receptor occupancy was investigated in a recent PET study; occupancy of dopamine D2 receptors corresponded to the doses of aripiprazole, but the changes in dopamine synthesis capacity were not significant, nor was the relation between dopamine D2 receptor occupancy and these changes [41]. It is well-known that only $2 \mathrm{mg}$ of aripiprazole would occupy approximately $70 \%$ of D2 receptors $[42,43]$. Therefore, our results may suggest that aripiprazole dose should not exceed doses usually needed in MDD treatment in routine practice. Moreover, we have to also consider that higher prevalence of CYP2D6*10 allele, which decreases CYP2D6 enzyme activity, in Asians than in Western population $[26,44]$.

Our study has clear shortcomings for generalization of the findings. The study design was retrospective with blinding method and placebo. Natural course of the illness or outcome biases could not be excluded. Furthermore, sample size power was proper by statistical calculation but absolute size should not be adequate for clinical translation. Antidepressants were not controlled. 
Assessment and treatment outcome biases by retrospective design should be also considered. Moreover, recall bias for diagnosis of MDDM should also be considered. Finally, the 2-month study duration was not adequate for proper evaluation of MDDM patients.

In conclusion, our results have clearly shown the effectiveness and tolerability of aripiprazole augmentation for MDDM patients in routine practice. The present study warrants subsequent, adequately-powered, RCTs for generalizability near future.

\section{- Conflicts of Interest}

This study was funded by a grant from Korea Otsuka Pharmaceutical. The funding source has not involved in any activities such as study protocol development, study design, data collection, data interpretation, and paper writing.

\section{Author Contributions}

Conceptualization: Chi-Un Pae, Changsu Han. Protocol development: Chi-Un Pae. Draft writing: Chi-Un Pae, Changsu Han. Intellectual comments and critics on the content: Sheng-Min Wang, Won-Myong Bahk, Soo-Jung Lee, Ashwin A. Patkar, Prakash S. Masand. Data acquisition: Chi-Un Pae, Changsu Han. Data analysis: Chi-Un Pae, Changsu Han, Sheng-Min Wang, Ashwin A. Patkar, Prakash S. Masand.

\section{ORCID}

Changsu Han https://orcid.org/0000-0002-4021-8907 Sheng-Min Wang https://orcid.org/0000-0003-2521-1413 Won-Myong Bahk https://orcid.org/0000-0002-3882-7930 Soo-Jung Lee https://orcid.org/0000-0002-1299-5266 Ashwin A. Patkar https://orcid.org/0000-0003-0484-1301 Prakash S. Masand https://orcid.org/0000-0003-1973-9449 Chi-Un Pae https://orcid.org/0000-0003-1632-4248

\section{REFERENCES}

1. Rush AJ. STAR*D: what have we learned? Am J PSychiatry 2007;164:201-204.

2. Woo YS, Shim IH, Lee SY, Lee DB, Kim MD, Jung YE, et al. Dose trends of aripiprazole from 2004 to 2014 in psychiatric inpatients in Korea. Clin Psychopharmacol Neurosci 2017;15:177-180.

3. Han C, Wang SM, Bahk WM, Lee SJ, Patkar AA, Masand PS, et al. A pharmacogenomic-based antidepressant treatment for patients with major depressive disorder: results from an 8-week, randomized, single-blinded clinical trial. Clin Psychopharmacol Neurosci 2018;16:469-480.

4. Dold M, Bartova L, Souery D, Mendlewicz J, Serretti A, Porcelli S, et al. Clinical characteristics and treatment outcomes of patients with major depressive disorder and comorbid anxiety disorders - results from a European multicenter study. J Psychiatr Res 2017;91:1-13.

5. McIntyre RS, Soczynska JK, Cha DS, Woldeyohannes HO, Dale RS, Alsuwaidan MT, et al. The prevalence and illness characteristics of DSM-5-defined "mixed feature specifier" in adults with major depressive disorder and bipolar disorder: results from the International Mood Disorders Collaborative Project. J Affect Disord 2015;172:259-264.

6. Vázquez GH, Lolich M, Cabrera C, Jokic R, Kolar D, Tondo L, et al. Mixed symptoms in major depressive and bipolar disorders: a systematic review. J Affect Disord 2018;225:756-760.

7. Hong N, Bahk WM, Yoon BH, Min KJ, Shin YC, Jon DI. Improving the screening instrument of bipolar spectrum disorders: weighted Korean version of the mood disorder questionnaire. Clin Psychopharmacol Neurosci 2018;16: 333-338.

8. Wang SM, Han C, Lee SJ, Jun TY, Patkar AA, Masand PS, et al. Second generation antipsychotics in the treatment of major depressive disorder: an update. Chonnam Med J 2016;52: 159-172.

9. Seo JS, Bahk WM, Wang HR, Woo YS, Park YM, Jeong JH, et al. Korean Medication Algorithm for Depressive Disorders 2017: third revision. Clin Psychopharmacol Neurosci 2018; 16:67-87.

10. Pae CU, Serretti A, Patkar AA, Masand PS. Aripiprazole in the treatment of depressive and anxiety disorders: a review of current evidence. CNS Drugs 2008;22:367-388.

11. Nelson JC, Mankoski R, Baker RA, Carlson BX, Eudicone JM, Pikalov A, et al. Effects of aripiprazole adjunctive to standard antidepressant treatment on the core symptoms of depression: a post-hoc, pooled analysis of two large, placebo-controlled studies. J Affect Disord 2010;120:133-140.

12. Trivedi MH, Thase ME, Fava M, Nelson CJ, Yang H, Qi Y, et al. Adjunctive aripiprazole in major depressive disorder: analysis of efficacy and safety in patients with anxious and atypical features. J Clin Psychiatry 2008;69:1928-1936.

13. Stewart TD, Hatch A, Largay K, Sheehan JJ, Marler SV, Berman $\mathrm{RM}$, et al. Effect of symptom severity on efficacy and safety of aripiprazole adjunctive to antidepressant monotherapy in major depressive disorder: a pooled analysis. I Affect Disord 2014; 162:20-25.

14. Pae CU, Patkar AA. Clinical issues in use of atypical antipsychotics for depressed patients. CNS Drugs 2013;27 Suppl 1:S39-S45.

15. Strejilevich SA, Teitelbaum J, Martino DJ, Quiroz D, Kapczinski F. Dopamine sudden depletion as a model for mixed depression. Med Hypotheses 2012;78:107-112.

16. Conway CR, Chibnall JT, Cumming P, Mintun MA, Gebara 
MA, Perantie DC, et al. Antidepressant response to aripiprazole augmentation associated with enhanced FDOPA utilization in striatum: a preliminary PET study. Psychiatry Res 2014;221:231-239.

17. American Psychiatric Association. Diagnostic and statistical manual of mental disorders. 5th ed. Arlington, VA:American Psychiatric Publishing;2013.

18. Montgomery SA, Asberg M. A new depression scale designed to be sensitive to change. BrJ Psychiatry 1979;134:382-389.

19. Young RC, Biggs JT, Ziegler VE, Meyer DA. A rating scale for mania: reliability, validity and sensitivity. $\mathrm{Br} / \mathrm{Psychiatry}$ 1978;133:429-435.

20. Sheehan KH, Sheehan DV. Assessing treatment effects in clinical trials with the discan metric of the Sheehan Disability Scale. Int Clin Psychopharmacol 2008;23:70-83.

21. Guy W. ECDEU assessment manual for psychopharmacology. Rockville, MD:U.S. Department of Health Education and Welfare; 1976.

22. Stahl SM, Morrissette DA, Faedda G, Fava M, Goldberg JF, Keck PE, et al. Guidelines for the recognition and management of mixed depression. CNS Spectr 2017;22:203-219.

23. Bandelow B, Baldwin DS, Dolberg OT, Andersen HF, Stein DJ. What is the threshold for symptomatic response and remission for major depressive disorder, panic disorder, social anxiety disorder, and generalized anxiety disorder? I Clin Psychiatry 2006;67:1428-1434.

24. Takeshima M. Treating mixed mania/hypomania: a review and synthesis of the evidence. CNS Spectr 2017;22:177-185.

25. Suppes T, Eudicone J, McQuade R, Pikalov A 3rd, Carlson B. Efficacy and safety of aripiprazole in subpopulations with acute manic or mixed episodes of bipolar I disorder. I Affect Disord 2008;107:145-154.

26. Kamijima K, Higuchi T, Ishigooka J, Ohmori T, Ozaki N, Kanba S, et al. Aripiprazole augmentation to antidepressant therapy in Japanese patients with major depressive disorder: a randomized, double-blind, placebo-controlled study (ADMIRE study). J Affect Disord 2013;151:899-905.

27. Kamijima K, Yasuda M, Yamamura K, Fukuta Y. Real-world effectiveness and safety of aripiprazole augmentation therapy in patients with major depressive disorder. Curr Med Res Opin 2018;34:2105-2112.

28. Ozaki N, Otsubo T, Kato M, Higuchi T, Ono H, Kamijima K; ADMIRE Study Group. Efficacy of aripiprazole augmentation in Japanese patients with major depressive disorder: a subgroup analysis and Montgomery-Åsberg Depression Rating Scale and Hamilton Rating Scale for Depression item analyses of the Aripiprazole Depression Multicenter Efficacy study. Psychiatry Clin Neurosci 2015;69:34-42.

29. Berman RM, Fava M, Thase ME, Trivedi MH, Swanink R, McQuade RD, et al. Aripiprazole augmentation in major depressive disorder: a double-blind, placebo-controlled study in patients with inadequate response to antidepressants. CNS Spectr 2009; 14:197-206.
30. Berman RM, Marcus RN, Swanink R, McQuade RD, Carson $\mathrm{WH}$, Corey-Lisle PK, et al. The efficacy and safety of aripiprazole as adjunctive therapy in major depressive disorder: a multicenter, randomized, double-blind, placebo-controlled study. J Clin Psychiatry 2007;68:843-853.

31. Marcus RN, McQuade RD, Carson WH, Hennicken D, Fava $\mathrm{M}$, Simon JS, et al. The efficacy and safety of aripiprazole as adjunctive therapy in major depressive disorder: a second multicenter, randomized, double-blind, placebo-controlled study. J Clin Psychopharmacol 2008;28:156-165.

32. Jon DI, Kim DH, Seo HJ, Kwon YJ, Kim MD, Yang JC, et al. Augmentation of aripiprazole for depressed patients with an inadequate response to antidepressant treatment: a 6-week prospective, open-label, multicenter study. Clin Neuropharmacol 2013;36:157-161.

33. Pae CU, Jeon HJ, Lee BC, Seo HJ, Kim SG, Park EJ, et al. Aripiprazole augmentation for treatment of patients with chronic or recurrent major depressive disorder: a 12-week prospective open-label multicentre study. Int Clin Psychopharmacol 2013;28:322-329.

34. Han C, Wang SM, Seo HJ, Lee BC, Jeon HJ, Kim W, et al. Aripiprazole augmentation, antidepressant combination or switching therapy in patients with major depressive disorder who are partial- or non-responsive to current antidepressants: a multi-center, naturalistic study. J Psychiatr Res 2014;49:75-82.

35. Han C, Wang SM, Kwak KP, Won WY, Lee H, Chang CM, et al. Aripiprazole augmentation versus antidepressant switching for patients with major depressive disorder: a 6-week, randomized, rater-blinded, prospective study. J Psychiatr Res 2015;66-67:84-94.

36. Pae CU, Wang SM, Han C, Lee SJ, Patkar AA, Masand PS. Aripiprazole augmentation for major depressive disorder: dosing patterns in a naturalistic treatment setting. Int Clin Psychopharmacol 2014;29:116-119.

37. Kelly T, Lieberman DZ. The utility of low-dose aripiprazole for the treatment of bipolar I/ and bipolar NOS depression. J Clin Psychopharmacol 2017;37:99-101.

38. Thase ME, Jonas A, Khan A, Bowden CL, Wu X, McQuade RD, et al. Aripiprazole monotherapy in nonpsychotic bipolar / depression: results of 2 randomized, placebo-controlled studies. J Clin Psychopharmacol 2008;28:13-20.

39. Suppes T, Silva R, Cucchiaro J, Mao Y, Targum S, Streicher C, et al. Lurasidone for the treatment of major depressive disorder with mixed features: a randomized, double-blind, placebo-controlled study. Am J Psychiatry 2016;173:400-407.

40. Suppes T, Kroger H, Pikalov A, Loebel A. Lurasidone adjunctive with lithium or valproate for bipolar depression: a placebo-controlled trial utilizing prospective and retrospective enrolment cohorts. J Psychiatr Res 2016;78:86-93.

41. Ito H, Takano H, Arakawa R, Takahashi H, Kodaka F, Takahata K, et al. Effects of dopamine D2 receptor partial agonist antipsychotic aripiprazole on dopamine synthesis in human brain measured by PET with L-[ $\beta-11 C] D O P A$. PLoS One 
2012; 7:e46488.

42. Sparshatt A, Taylor D, Patel MX, Kapur S. A systematic review of aripiprazole--dose, plasma concentration, receptor occupancy, and response: implications for therapeutic drug monitoring. J Clin Psychiatry 2010;71:1447-1456.

43. Yokoi F, Gründer G, Biziere K, Stephane M, Dogan AS, Dannals RF, et al. Dopamine D2 and D3 receptor occupancy in normal humans treated with the antipsychotic drug aripi- prazole (OPC 14597): a study using positron emission tomography and [11C]raclopride. Neuropsychopharmacology 2002;27:248-259.

44. Ji L, Pan S, Marti-Jaun J, Hänseler E, Rentsch K, Hersberger M. Single-step assays to analyze CYP2D6 gene polymorphisms in Asians: allele frequencies and a novel ${ }^{*} 14 B$ allele in mainland Chinese. Clin Chem 2002;48:983-988. 